\title{
The Plant Endoplasmic Reticulum: A Dynamic Network of Interconnected Membranes
}

\section{Federica Brandizzi}

MSU Plant Research Lab - Michigan State University, East Lansing, Michigan, United States

The endoplasmic reticulum (ER) is a conserved organelle of eukaryotic cells that exhibits a formidable pleiomorphic structure. The ER synthesizes one third of the cellular proteome as well as essential lipids. It is also the site for $\mathrm{N}$-glycosylation and quality control of secretory proteins that populate the cell or are transported to the extracellular space allowing the cell to communicate with the external environment ${ }^{1}$. Defects in ER functions can be lethal, and over the last decade, it has become evident that the function and morphology of the ER are interconnected. Furthermore, because of its biosynthetic role, the ER is a suitable organelle for high-capacity production of endogenous and heterologous eukaryotic proteins. Therefore, understanding how the ER establishes and maintains its morphology is not only important in basic cell biology, it can also inform biotechnology efforts for the optimization of eukaryotic cells as bioreactors. It is generally believed that there is only one ER per cell. Remarkably, this organelle pervades the entire cell. The ER assumes the shape of a network of tubules that are continuously remodeled into other tubules and flattened cisternae. Owing to the presence of the large central vacuole, in plant cells the ER is mainly distributed to the cell cortex and transverses the central vacuole through invaginations of the vacuolar membrane. The ER interacts with other organelles and it is attached to other membranes, including the plasma membrane. The understanding of the mechanisms underpinning ER morphology in plant cells is still in its infancy. However, we know that maintenance of the dynamic structure of the ER is critical to cell growth, as demonstrated by the characterization of loss-of-function mutations shown to negatively affect the ER morphology and concomitantly organ growth. In this talk, we will describe our fluorescent protein-based approaches to identify and characterize the proteins that control the dynamic morphology of the plant ER in the context of organ growth.

\section{References}

1. Stefano, G. \& Brandizzi, F. Advances in Plant ER Architecture and Dynamics. Plant Physiol 176, 178186 (2018). 